live in a state of inactivity for years. It is this phase of the subject that should make us hold every case of syphilis sub judice for years, at least seven. I do not advise active treatment for that period, but after the first three years of active treatment with mercury, a mixed treatment during a month twice a year for three years more, will serve to prevent any further destructive action in ordinary cases.

The purpose is to keep the disease and its manifestations subjected to remedies. The absurd belief entertained by many, that mercury produces paralysis and locomotor ataxia, has been so often controverted in my experience that I would not discuss this feature of the subject, were it not for the grave injury which it is doing. I am treating now patients with locomotor ataxia and in nearly every form of paralysis, to whom I am giving maximum doses of mercury with the best results. This has been my experience for a number of years. My observation has been that nearly all the cases of syphilitic paralysis have been due to treatment for too short a period, or that there never had been a full mercurial effect produced. I feel quite sure that I shall be sustained in this statement by every close observer of the phenomena of this disease. In my discussion of the proper treatment I assume that all other indications have been filled; that the patients have lived soberly and carried out intelligent hygienic rules for their guidance. Had the theory that mercury produced paralysis been verified, the syphilitic paralytics whom I have treated during the last twenty years would have succumbed to the mercury which I gave, as I am very generous in my prescriptions. It seems to me to be a logical conclusion that syphilis is due to a protozoon, a microörganism, a living entity. The whole theory of treatment is to kill this living germ without killing the man in whom it is existing. So far progress in medicine has not discovered anything better or so good as mercury with which to combat its injuries and to limit its existence.

As the objective of all treatment is to poison the specific germ, and at the same time render the patient unfit for its abode, it follows that the greater the quantity of mercury that can be introduced, without danger, the better the protection given. The action of mercury is directly as a poison and indirectly as a tonic, in other words, when the microörganisms are killed or weakened, the system will re-assert its normal vigor.

If syphilis is due, as is now believed by scientific men everywhere, to a protozoon, it would account for many cases of attenuated innoculations which do not give the characteristic symptoms and features of the disease that we are accustomed to recognize and which render our diagnosis difficult.

Many years since, Kassowitz, of Vienna, who had charge of the hospital for syphilitic women and children, stated that he frequently met with women married to men in the later stages of syphilis (and who had been previously healthy) cachectic and devitalized, without showing any uther symptoms of a specific contagion, and yet who failed to improve under any other than specific treatment. He believed that in many cases a married life between a man with tertiary syphilis and a perfectly healthy woman would be followed by the loss of strength and vitality to the latter.

While my own experience does not confirm his in many particulars, I have seen so many suggestive cases that I feel as if I were leaning to virtue's sidə when I discourage marriage in all syphilitics who had not been treated for many years and who were not leading a life conforming to the laws of hygiene. There is an obligation on the medical profession to see to it that the progenitors of future generations do not hand down diseased bodies and minds. This responsibility should not be ignored, however great the temptation to popularize ourselves by pursuing a different course. Let us guard the race by establishing a quarantine against all contagious diseases. To prevent the crime of the marriage of syphilitics, in the earlier stages, would it not be commendabie to have a law requiring a health certinicate from a board of examiners accompany every marriage license?

Very candidly and frankly, I see no hope for the syphilitic at present except in mercury and the iodids, although I fully believe that the day is not far off when some virus will be used for innoculation, which will relieve mankind of the frightful results of this malady. Experiments with horse serum are in the right direction.

\section{THE NEW SURGICAL SPLINTING.}

Read in the Section on Surgery and Anatomy, at the Forty-sixth Annual Meeting of the American Medical Association, at Baltimore. Md., May $\mathbf{7}-10.1895$.

BY EDWARD A. TRACY M.D.

MEMBER OF THE AMERICAN MEDICAL ASSOCIATION. BOSTON, MASS.

Two years ago at the Milwaukee meeting of the Association, I had the honor to read a short paper entitled "A New Material for Surgical Splints and Spinal Jackets, with a Method for Applying It." A few months later I read before the Pan-American Medical Congress held in Washington, a paper entitled "A Brief Splint-Technology for Surgeons," in which has been outlined a scientific system of surgical splinting. As a small contribution to the filling in of this outline I shall present to-day four new splint forms and report cases illustrating their use. Before presenting them to your consideration, permit me to speak for a moment of the material used in their construction. It is composed of crushed woodfiber, rolled in a rugose fashion, and in which a fabric is incorporated during the rolling process. The material is the result of original experimental work, having for its object the attainment of a moldable material that would comply with the requirements of a scientific method of surgical splinting. The experimentation was done by me for the most part in a pulp mill, the use of which was courteously placed at my disposal by my kind friend, Mr. Charles $\mathrm{H}$. Fish, of Manchester, N.H. The material and method of splinting as presented to you, represents the practical solutions of the several problems met by me during the progress of my work, problems relating to the toughness, plasticity, rigidity and technical manipulation of a splint material having for its basis woodfiber. None the less do I believe that the work presented to your consideration will seem admirable for its simplicity.

Having considered the splint material, allow me to describe briefly the ordinary manner by which I make from it a splint. The first thing to be done is to cut the splint blank from a sheet of the material. The outline for this blank can readily be gotten for the ordinary leg and arm splints, by simply placing a sheet of the material against the limb and pencil- 
ing the outline directly on it. In other cases a pattern of the splint blank can be made by cutting a piece of paper the proper shape to enfold accurately the part it is desired to splint, and then laying this paper pattern on a sheet of the material and penciling the outline of the pattern on it. By following the outline, the splint blank can be cut from the material by means of a jack-knife or a pair of shears. A suitably shaped blank having been cut from the material, it should be moistened so as to become plastic or moldable. The moistening can be done with water, but it is better to moisten the blank with a solution of potassium silicate. The ordinary solution sold for surgeons' use and further diluted with half its bulk of water I find serves admirably. This solution adds considerably to the rigidity of the splint. It is also an antiseptic. The moistening is best done by painting the solution first on one side of the splint blank and then on the other side, and so on two or three times, using for the purpose an ordinary flat paste brush. The splint material will be seen to absorb the moisture, and after a minute or two will become quite plastic or moldable. The blank should then be applied directly to the limb and a roller bandage snugly applied over it so as to make it conform to the shape of the part we intend to splint. The blank should be carefully removed and dried over a kitchen fire, gas stove, gas jet or other sufficient source of heat. While it is drying the splint should be watched and helped, by holding it properly, to retain the right shape. Before it is completely dry, it should be applied to the limb for a moment and, if need be, corrections made, before complete drying permanently fixes its form. The drying of the ordinary splints averages from ten to fifteen minutes. A single layer of merino (to be had in most households from an old garment of underwear) or a few layers of gauze is all that should be placed between the splint and the skin when applying the splint to the case for which it has been made.

In many cases of simple joint fixation, if the proper thickness of the splint material be used, (it is manufactured in sheets of three different sizes by the American Wood Pulp Company, of Boston.) the splint blank can be molded over the parts and a bandage applied directly and the splint allowed to dry on the limb without removing it. The four splint forms which I shall here describe have been chosen because of their utility, simplicity, and to suggest to you the scope of the new splinting. I am sure that the practitioner who devotes a little attention to them, or even to the first splint form to be described, will be rewarded with the result. These forms can serve as an introduction to the use of my splint material and after familiarizing himself with its characteristics the practitioner will be enabled to work it into such forms as the indications met with in any given case may call for. This mastery of the material is readily attained by the careful practitioner. But should he not care to push his conquest of it further than these simple splint forms, I repeat, the time so spent will repay him, and he will be surprised at the readiness with which he can get an efficient fixation of the knee, elbow, ribs (as much as the anatomical conditions of the ribs permit) or hip. The esthetic feature of the new splinting, both of color and form, and its comfort to the wearer by reason of its lightness, I shall but mention; they are best appeciated by the patient.
IATERO-POSTERIOR KNEE SPLINT, FOR FIXATION OF THE KNEE-JOINT.

The pattern for this splint should be cut so as to embrace the posterior and one of the lateral aspects (preferably the inner) of the thigh and leg, extending from well up the thigh to a few inches from the ankle. Following this pattern for a guide, the splint blank should be cut from a sheet of the splint material having a thickness of about $2 \mathrm{~mm}$. The splint blank should be moistened, molded and dried in the usual manner before described. This splint well illustrates the mechanical principle by which the breaking strain of any piece of flat material is greatly increased as it is made to assume the tubular form, or by having a flange in its form. A piece of the splint material of the thickness described, molded over the posterior aspect of the limb only, could not be expected to serve for the fixation of a joint con-

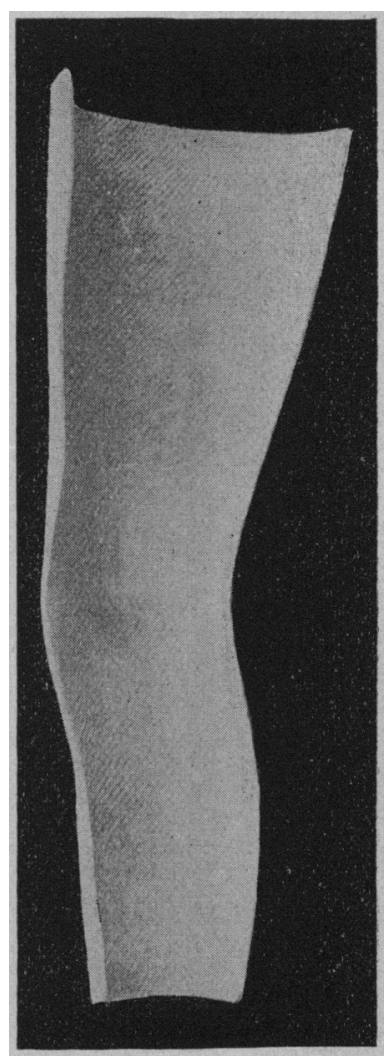

FIG. 1.-A Latero-posterior knee splint, left.

trolled by such powerful muscles as in the knee. But by simply widening the blank so that it can be molded over a lateral aspect of the limb as well as the posterior aspect, the needful element of tensile strength is increased in far greater proportion than to the extra amount of the material employed. Another advantage is that the splint so molded and applied to the limb can not move from side to side; its flange molded over the side of the limb prevents this; it therefore gives a fixation superior to any posterior or ham splint. This splint is illustrated in Fig. 1, which is taken from a photograph of one molded ou a patient (J.W., aged 53 ) by me at the Boston City Hospital (by courtesy of Dr. Thorndike). The case was acute exacerbation of a chronic synovitis of the left knee. The splint was worn three weeks with satisfactory resuits. At the same hos- 
pital I treated a young man with an acute synovitis of the right knee, of traumatic origin. There was considerable effusion in to the joint. A similar splint was molded on the patient's limb and worn for eight days. The effusion and tenderness had then disappeared; the patient was ordered to use the limb carefully, and to return to his organ studies. He did so without any recurrence of the trouble.

AN ELBOW SPLINT (EXTERNAL) FOR FIXATION OF THE ELBOW JOINT.

This splint serves for fixation of elbow joint and as it does not impinge upon the internal aspect of the joint allows a ready inspection of the internal condyle without the necessity of removing the splint. It is of easy construction. A pattern of the splint blank is cut so as to embrace the flexed arm and forearm; the upper part from the fold of the axilla to that of the elbow, the lower part extending from the olecranon and fold of the elbow to the knuckles of the hands, the arm being semi-pronated. The pattern should be cut so as to fold over or embrace the ulnar side of the forearm and hand. Following this pattern for a guide, the splint blank should be cut from a sheet of the splint material having a thickness of about $2 \mathrm{~mm}$. It should then be

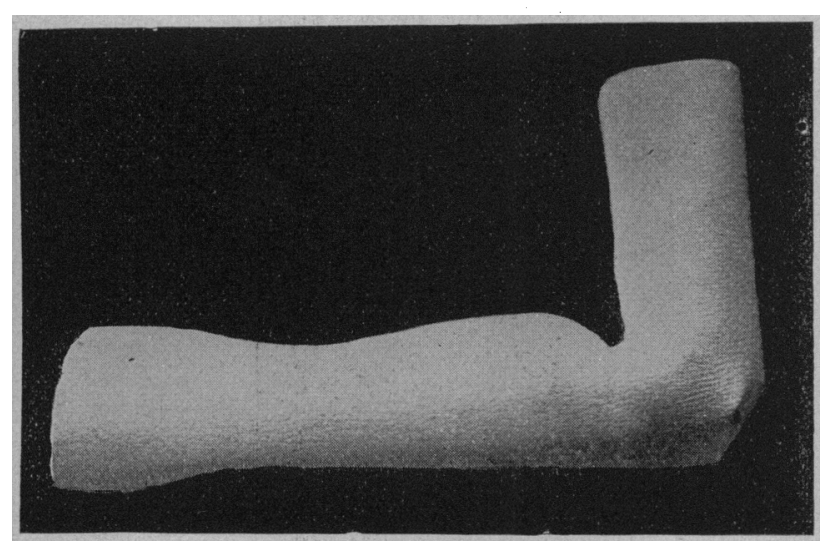

Fig. 2.-An external angular elbow splint; left arm. View is of the outer aspect.

moistened, molded and dried in the ordinary manner before described. The slight redundancy of material found near the olecranon when molding the blank should be pinched up between the fingers and bent over so as to lie against the splint. This splint is illustrated in Fig. 2, taken from a photograph of a splint molded by me on the left arm of Willie M., a boy who was brought to my office with a backward dislocation of the elbow and a fracture of the internal epicondyle, caused by a fall from a height of about five feet. I reduced the dislocation and molded the splint in accordance with the technique given above, and bandaged it to the arm, leaving the internal condyle exposed to sight. I then placed a cloth compress over the internal epicondyle and bandaged it so as to retain the fragment in its proper position. After six days the splint was removed daily so as to exercise the the joint with gentle passive motions, and after eleven days the splint was omitted entirely. On the twentieth day all motions of the joint were normal, except extension, which was slightly limited. The boy then disappeared for awhile, and when next examined, three months after, nuthing abnormal except a very slight obtundity of the internal epicondyle could be detected; all the motions were perfectly normal.

A similar splint was employed in the case of $A$. N., an old man treated by me at the Boston City Hospital (by courtesy of Dr. Thorndike). The case was a fracture of the right ulna at its middle third, caused by the kick of a horse. The fixation of the fragments was attained by this splint supplemented by a palmar splint extending from the elbow fold to the wrist. In seventeen days there was union; on the twenty-first day the angular splint was cut across below the angle of the elbow, so that it became a simple dorsal forearm splint, leaving the elbow joint free. For safety this splint was left on for eight days, and after twenty-nine days from the beginning of treatment the patient was discharged well, with normal mobility to the arm and forearm, and but a moderate callus. The indication in this case, complete immobilization of the ulna, was read-

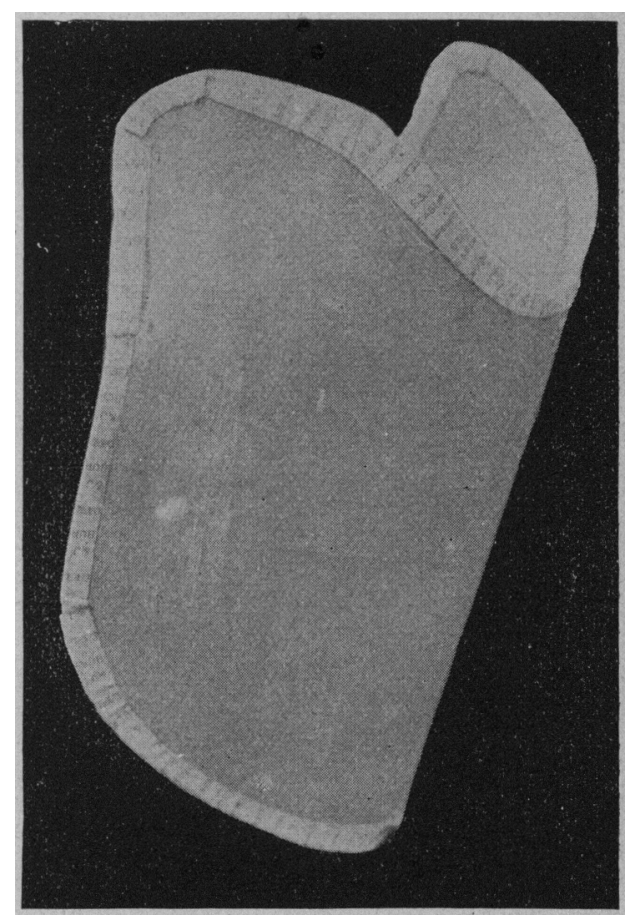

Fig. 3.-A splint used in a case of rib fracture; left. A front view of the splint.

ily met by fixation of the elbow joint and of the wrist joint by aid of the lateral angular splint and boxing in of the forearm by aid of the palmar piece from the elbow to the wrist. The result was considered excellent considering the age and condition of the patient (about 70 years, and with a double cataract and atheromatous arteries).

A SPLINT FOR RIB INJURIES.

This splint is a very simple one and readily constructed. It should be molded over the injured side of the chest, from an appropriately shaped splint blank. The blank varies in shape according to the situation of the injury and also to the fact of the chest being that of a male or female. These slight variations of shape need not be detailed, as they will readily suggest themselves for the case under treatment, when the principles governing the treatment are understood. Fig. 3 is from the photograph of a 
splint molded over the chest of Mrs. C., aged 58 years, treated hy me for fracture of the eighth and ninth left ribs. The splint was worn twenty-one days, being easily retained in position by a snugly applied swathe, to which was applied a shoulder strap consisting of a piece of cotton cloth held by safety pins, over the left shoulder. This method of treatment for the ordinary case of fractured ribs seems a rational one. I do not understand how adhesive plaster, or a simple swathe can protect the broken bones from external pressure that may occur at any time, and particularly when the patient sleeps. The rib splint, however, spanning the weakness in the chest wall and resting on the secure foundation of the sound ribs, prevents undue pressure from reaching the point of fracture. This, doubtless, explains the comfort with which the splint is worn. Moreover the indication to limit motion in the affected side is effectually met by a properly constructed splint molded so as to fit the half chest in the position it assumes on forced expiration. The splint thus made tends of itself to hold the half chest in that position, and its efficacy in this respect is perfected by the swathe with shoulder strap attached as described which maintains it in position.

\section{A HIP SPLINT.}

This splint furnishes a ready method for obtaining an efficient fixation of the hip joint without the extraneous aid of blacksmithing or special tools. A pattern should be cut so as to extend from an inch or two below the axilla to about the same distance below the knee. The posterior border of the pattern commencing at the upper border should follow near to the spinal column, and over the buttock down the posterior and inner surface of the thigh to the inferior border of the pattern below the knee. The anterior border of the pattern commencing at the superior border below the level of the axilla, should pass over the chest and belly near to the middle line of the body, striking off below the umbilicus to cross the lower third of Poupart's ligament and down the inner surface of the thigh to end at the inferior border below the knee. Following the pattern for a guide the splint blank should be cut from a sheet of the material having a thickness of $2 \mathrm{~mm}$. This splint blank should be moistened, molded and dried in the manner described above. Another blank should now be cut from a sheet of thinner material, and it should be moistened in like manner and molded over the other one so as to serve as a reinforcement. The two forms should be laid together as one, with a layer of liquid glue between them, and applied as one to the limb. While molding this splint the patient should be laid on his back with the limb extended in a line with the body. The moistened blank should be bent so as to embrace the affected thigh and side, and molded over the parts by means of a roller bandage. The splint should be finished by binding its border with strips of chamois skin fastened on with a cheap liquid glue. Immediately after binding the splint it can be applied to the patient, the glue setting as well with the splint in position. This splint should be properly bandaged to the body if we desire the maximum of hip fixation. This is done by taking a few tight turns around the hips with the roller bandage and then bringing the roller down so as to embrace, in figure 8 style, the upper portion of the affected thigh and the opposite hip. A bandage should then be applied from the lowest portion of the splint, embracing first the knee and thigh, then the hips and finally the chest. The bandaging can be prevented from slipping by pinning together the layers of bandage where they overlap. The splint illustrated in Fig. 4 is taken from a photograph of a splint molded by me on Cornelius O'H., a boy aged 8 , with hip joint disease of the left hip. Night cries and pain ceased shortly after its application. In three week the boy was enabled to be up and out by means of crutches and a raised shoe. After four months, tenderness on deep pressure over the capsule of the joint had somewhat diminished, but flexion and outward rotation were considerably impaired. The splint was worn for eleven months with no setback to the improvement of the patient. The parents of my patient then insisted on the withdrawal of all treatment, crutches, raised shoe, and splint. At that

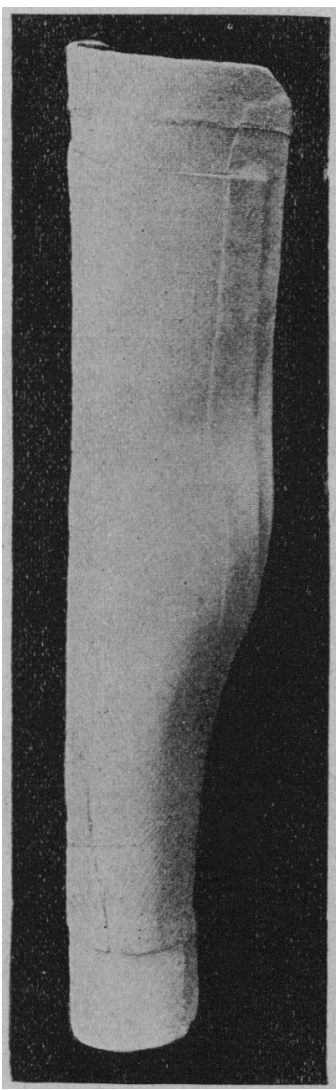

Fig. 4.-A Hip splint; left. View is of the posterior aspect.

tıme there was a slight limitation to flexion, the left gluteal fold was absent, and considerable atrophy of the left thigh present, though not as much as six months before. I warned the parents as to the probability of their being trouble in the joint, but their determination to have the boy use his limb remained unchanged. They promised me to watch for symptoms and on the first appearance of a limp or pain to resume treatment. There has been no recurrence and now (April 27), seven months since omission of treatment, upon examination I find his gait, walking and running, normal. No atrophy of thigh or leg. Gluteal fold present, no tenderness of hip joint. All motion except extreme flexion of the thigh normal. Upon extreme flexion the pelvis moves with the thigh bone, though no pain is felt. The boy has been attending school since January, and is as full of life on the streets as any of his comrades. [Jan. 14, 
1896 , over fifteen months since omission of treatment, the boy is as well as ever.]

It needs but a little consideration to understand the thorough fixation procured by this splint when correctly applied. A cursory examination will show that adduction, abduction, extension and flexion and their combination, circumduction, are out of the question with this splint in position. It is left only to consider the remaining motion of the hip joint-rotation. It is prevented by the grasp which the splint maintains on the knee, and also by its forward pressure on the great trochanter. The trochanter, it will be remembered, swings backward in an arc on outward rotation of the thigh.

In concluding my paper I will remark, that because of circumstances not controllable by me, the splint material has not been accessible to you all, and therefore you can not testify to the advantages of the new method of surgical splinting from personal experience with it. It gratifies me exceedingly, however, to be able to refer to Dr. George W. Gay, of the Boston City Hospital, who wrote in a note to me last November: "You may quote me as being favorably impressed with your surgical splinting after a considerable personal experience with it." The object of my work in surgical splinting is to emphasize the practicability of living up to the principle of surgical practice enunciated by Dr. H. O. Marcy: "The surgeon must make a splint to fit the limb and not the limb to the splint." I believe, now that the splint material is accessible to the general practitioner, that the method described, will result in the completion of a scientific system of surgical splinting that will do honor to our Assocration and our country.

Note. Since reading the above paper I have done considerable splinting, including cases of hip, knee, and ankle joint fixation, Collis' fracture, using only water as a moistener, and alloving the splint to dry in position on the limb. In several cases absolutely nothing was placed between the limb and the splint, and though the splint was worn for several weeks no excoriation of the skin occurred. The object of this note is to emphasize the simplicity of the technique now employed water alone being found essential, the drying of the splint off the limb, and the use of a silicate solution being unnecessary. 99 Broadway, Boston, Mass.

\section{SOME REMARKS ABOUT ASEPSIS IN MILI.} TARY SERVICE. Read at the Meeting of the National Association of Military Surgeons,
Buffalo, May, 1895.

BY LIEUT.-COL. EDWARD BOECKMANN.

ASSISTANTSURGEON-GENERAL OF THE MINNESOTA NATIONAL GUARD.

It is with some hesitancy that I venture to bring such a worn-out topic as asepsis before this distinguished assembly, which is represented by our foremost bacteriologists, our best surgeons and by men who, perhaps, daily have opportunity to rejoice in the good fruits of their aseptic efforts. But when I at the same time consider that the last word on asepsis has not yet by any means been spoken, that our views are subject to change and our opinions vary, that there yet prevails considerable difference and even uncertainty with regard to the value of the different means and apparatus of disinfection, that daily. partly through ignorance and partly through negligence, violence is done to the simple but strict laws of asepsis, and that we military surgeons, because of the peculiar and difficult conditions under which we must exercise the aseptic art in service, particularly should be conversant with the principles of asepsis.
I certainly need no apology for presenting that which at first sight appears to be A, B, C to us all.

Our surgical enemies, which all of us know so well, either through our practice or through our books, and which are distinguished by their ubiquity and numbers, less by their tenacity of life, we endeavor to keep at a distance in different ways; we destroy them, we remove them or we render them innocuous. Could we simply destruy them all this would be the most ideal procedure, but since this, unfortunately, is impracticable, we must in every case avail ourselves not only of the chemic means of disinfection, which are the proper germicides, but also of mechanical and chemic measures whose usefulness consists in the removal and the inhibition of the growth of microorganisms.

\section{MECHANICAL DISINFECTION.}

Since we can not boil our hands or put our patients in a steam sterilizer and since the chemic disinfectants can not sterilize an infected skin in such concentration as can be used in practice and in such time as is at our disposal it is clear and universally conceded that the crucial point in the sterilizing process of the operator's hands and the patient's field of operation must lie in the mechanical removal of the present and innumerable microörganisms in these situations. This can also be essentially accomplished by razor, nail-cleaner, water, soap, brush and towel, but any absolute surgical operation is hardly within possibility, as the surgical microörganisms not only are met with in the deeper epidermic layers, but also in the different adnexa of the skin. That disinfection which results from a careful mechanical treatment of the skin, has been made fractional by different authors, some putting it at a half, others at twothirds, while, as is well known, there are those who both on this and the other side of the Atlantic find in the mechanical operation the ideal in surgical sterilization of the epidermis. In my opinion we can not well express in definite fractions the aseptic value of mechanical disinfection, since in the first place in. dividual differences obtain in high degree and since in the second place mechanical disinfection is an art exercised by different operators in extremely varying degrees of efficiency. It is unfortunately not to be denied that those operators who really understand how to wash themselves surgically still are in the minority, which is deplorable, as I am one of the many who have arrived at the conclusion that mechanical disinfection can nearly approach surgical asepsis, if practised intelligently. We should certainly be spared the painful spectacle of seeing a surgeon omit to remove his finger rings and at the same time it is only a reasonable demand that the shirt sleeves are rolled up beyond the elbow. It is unquestionably proper to use a razor on the field of operation, not so much for the sake of the hair as for the skin, whose disinfection is greatly facilitated. The surgeon himself by rights does not shave his arms or hands, which would be a necessity if this process showed a sine qua non for the disinfection of the hair. The washing itself must be performed in a routine manner; first a general soaping and scrubbing, thereupon a special ditto of each part. The extensor surface of the arms is reached by flexion and not by extended pronation or supination. Special attention is given the fiuger ends, the nails and the folds between the fingers. The more changes of 